\title{
Edad, crecimiento y mortalidad de la almeja de sifón, Panopea globosa (Bivalvia: Hiatellidae) en la región central del Golfo de California, México
}

\author{
Age, growth and mortality of the cortes geoduck, Panopea globosa (Bivalvia: Hiatellidae) \\ in the central Gulf of California, Mexico \\ Gustavo Cortez-Lucero', José Alfredo Arreola-Lizárraga ${ }^{1}$, \\ Jorge Chávez-Villalba ${ }^{1}$ y Eugenio Alberto Aragón-Noriega ${ }^{1}$
}

${ }^{1}$ Centro de Investigaciones Biológicas del Noroeste, Unidad Sonora, Km 2.35 Camino al Tular, Estero Bacochibampo, Guaymas, Sonora 85454, México. aaragon04@cibnor.mx

\begin{abstract}
For the first time, this paper describes the age, growth and mortality of the cortes geoduck Panopea globosa in the Gulf of California. This study was motivated because of the limited knowledge regarding biological aspects of this species in Mexico. Commercial catches of geoduck Panopea generosa and $P$. globosa started along the Baja California coast in 2002, nowadays catches are 1200 ton, similar to those in Washington, USA and British Columbia, Canada, when fisheries started in 1970 and 1976, respectively. The objective of this study was to know the age, growth and mortality of $P$. globosa. Geoducks were collected from November 2008 through to February 2010, in the central eastern coast of the Gulf of California (Bahia del Sol, Sonora, Mexico). Age was established using the 'acetate peel' method and von Bertalanffy growth parameter was estimated as $L_{\infty}=122.86 \mathrm{~mm}, k=0.33$ and $t_{o}=-0.2$. Mortality according to each method ranged from 0.79 to 0.546 . Ages varied from 2 to 27 years old but the mode was $10 \mathrm{y}$. Average size $( \pm \mathrm{IC} \alpha 0,05)$ was $109.8 \pm 3 \mathrm{~mm}$ shell length. The growth parameter and mortality rates are similar to those found in young organisms of congener species. For these reasons the conclusion is that according to our findings, this stock is a recently established population in response to a favorable environmental event.
\end{abstract}

Key words: Fisheries, crossdating, growth, mortality

Resumen.- Debido a la carencia de conocimiento biológico básico de la almeja sifón Panopea globosa, en México, este estudio describe por primera vez la edad y crecimiento de esta especie obtenida desde el Golfo de California. La pesca comercial de almejas sifones (Panopea generosa y P. globosa) comenzó en Baja California en el 2002, con volúmenes de captura de 1200 ton a la fecha, lo cual es similar a la pesquería de este grupo de almejas en otros países (Washington, Estados Unidos y la Columbia Británica, Canadá) aun cuando la pesquería se inició en los años 70s. Este estudio se realizó con el objetivo de evaluar edad, crecimiento y mortalidad de organismos de $P$. globosa recolectados en la costa centro oriental del Golfo de California (Bahía del Sol, Sonora, México) entre noviembre 2008 y febrero 2010. La edad se determinó mediante el método de conteo de bandas de crecimiento en cortes transversales de la concha que es copiado en papel de acetato y ajustando el modelo de crecimiento de von Bertalanffy. La longitud asintótica fue de $122,86 \mathrm{~mm}$ con una tasa de crecimiento (k) de 0,33. La mortalidad se estimó con tres diferentes métodos. La mortalidad natural estimada fue de 0,079 a 0,546 según el método utilizado. El intervalo de edad estimado fue de 2-27 años, pero con mayor representatividad de los individuos de 10 años. La longitud promedio $( \pm I C \propto 0,05)$ de la concha fue de 109,8 $\pm 3 \mathrm{~mm}$. Los parámetros de crecimiento y mortalidad coinciden con los obtenidos en organismos jóvenes de otras especies del género, por lo que concluimos que nuestros resultados aportan evidencia de que la población de la parte central del Golfo de California es de reciente asentamiento en respuesta a un evento ambiental favorable.

Palabras clave: Pesquerías, datación cruzada, crecimiento, mortalidad 


\section{INTRODUCCIÓN}

Las almejas de sifón del Pacífico Panopea spp., ha estado bajo explotación pesquera a partir de los años 70s; desde 1970 en Washington, Estados Unidos, y a partir de 1976 en Columbia Británica, Canadá, donde fue valorada en aproximadamente 35 millones de dólares en 2006 (Khan 2006). A partir de 2002 se inició en México la pesca comercial de almeja de sifón Panopea generosa (Adams, 1850) en la costa del Pacífico de la península de Baja California y P. globosa (Dall, 1898) en el Golfo de California.

En particular los estudios sobre la almeja de sifón en México indican que Panopea globosa en el Golfo de California se han enfocado al conocimiento de su ciclo reproductivo y esta especie presenta su pico reproductivo entre enero y febrero cuando la temperatura superficial del mar se encuentra en aproximadamente $18^{\circ} \mathrm{C}$ (AragónNoriega et al. 2007); asimismo un análisis comparativo de los ciclos reproductivos de $P$. globosa de México con $P$. generosa de Canadá y P. zelandica (Quoy \& Gaimard, 1835) de Nueva Zelanda, mostró que la temperatura del agua es un factor clave para el éxito reproductivo de las almejas de este género (Arámbula-Pujol et al. 2008) y se ha aportado evidencia de, la relación del desarrollo larval de $P$. globosa con el pico de máxima producción de productividad primaria en el Alto Golfo de California que sostiene la hipótesis sobre acoplamiento reproductivo al ambiente (Calderón-Aguilera et al. 2010b). Sin embargo, se desconoce la estructura de edades y la tasa de crecimiento de la población, así como los parámetros de crecimiento individual. Este conocimiento es esencial para abordar la base biológica y la idoneidad de tamaño mínimo de captura permisible como una estrategia de gestión y aprovechamiento sostenible (Pauly 1996, Tijaro-Rojas \& Salgado-Cabrera 2007, Mora et al. 2009). El manejo pesquero de $P$. globosa se ha basado en el establecimiento de la longitud de mínima de captura y cuota de extracción por banco susceptible de pesca, pero sin el conocimiento biológico correspondiente. Es importante considerar que los estudios para estimar la biomasa y mortalidad por pesca, se realizan a partir de modelos basados en la estructura de edad (Bradbury \& Tagart 2000) la estimación de los parámetros de crecimiento requiere estimaciones precisas de edad. El método de contar los incrementos de líneas internas en cortes transversales de la concha, utilizando la técnica de papel de acetato, ha demostrado ser efectivo para la determinación de la edad de estos bivalvos longevos (> 10 años) y ha sido validado por varios autores (e.g., Shaul \& Goodwin 1982, Sloan \& Robinson 1984, Morsan \& Ciocco 2004, Black et al. 2008, Calderón-Aguilera et al. 2010a).

La información biológica y ecológica específica sirve para establecer puntos de referencia que orienten el manejo pesquero. Esto es especialmente importante considerando que se trata de especies que de acuerdo con las investigaciones en $P$. generosa, se caracterizan en general por ser muy longevas, tener bajas tasas de reclutamiento y crecimiento poblacional lento, apreciable sólo a largo plazo. De acuerdo con Orensanz et al. (2004), la explotación de especies con dichas características imponen problemas al manejo pesquero, especialmente si se conoce poco de la dinámica poblacional. Basado en lo anterior, el presente estudio tuvo como objetivos determinar la edad, los parámetros de crecimiento individual y mortalidad de Panopea globosa, en la región central del Golfo de California y adicionalmente, se probó la hipótesis del efecto de la temperatura del agua de mar sobre el crecimiento de la almeja de sifón en esta región.

\section{Materiales y MÉTOdos}

\section{Área de ESTUdio y MUESTREO}

La edad y el crecimiento de Panopea globosa fueron investigados realizando muestreos en la Bahía del Sol ubicada en la región central de la costa oriental del Golfo de California y localizada a los $27^{\circ} 53^{\prime} \mathrm{N}-110^{\circ} 41^{\prime} \mathrm{W}$ y $27^{\circ} 53^{`} \mathrm{~N}-110^{\circ} 40^{\prime} \mathrm{W}$ (Fig. 1). Los datos provienen de organismos recolectados cada mes desde noviembre 2008 hasta febrero del 2010 a una profundidad de entre 15 a 25 m. La captura se realizó por buceo semi autónomo con la ayuda de una motobomba que arrojaba agua a presión a través de una manguera de plástico de $50 \mathrm{~m}$ de largo y 5 $\mathrm{cm}$ de diámetro. Este método facilita la recolecta sin dañar a los organismos, el buzo localiza el sifón saliente o la marca de los orificios de un sifón contraído de la almeja, coloca la salida de agua de la manguera junto a la marca o sifón y se libera el flujo de agua a presión que saca a la almeja del sustrato ${ }^{1}$.

\section{TEMPERATURA SUPERFICIAL DEL MAR}

Los datos de temperatura superficial del mar (TSM) están disponibles en el sitio Web de la agencia estadounidense de administración nacional de océano y atmósfera $\left(\mathrm{NOAA}^{2}\right)$. Se obtuvo también información sobre el Índice

\footnotetext{
${ }^{1}$ Extracción de la almeja sifón, generado en la Asociación Canadiense de Pescadores, a través de un video disponible en <http:// www.geoduck.org/english-marketing-video_en.html>

${ }^{2}$ NOAA/ National Weather Service. National Centers for Environmental Prediction. Climate Prediction Center, Camp Springs, Maryland, Climate Prediction Center Web Team <http://www.emc.ncep.noaa.gov/research/cmb/sst_analysis/\#_cch2_1007146782>
} 


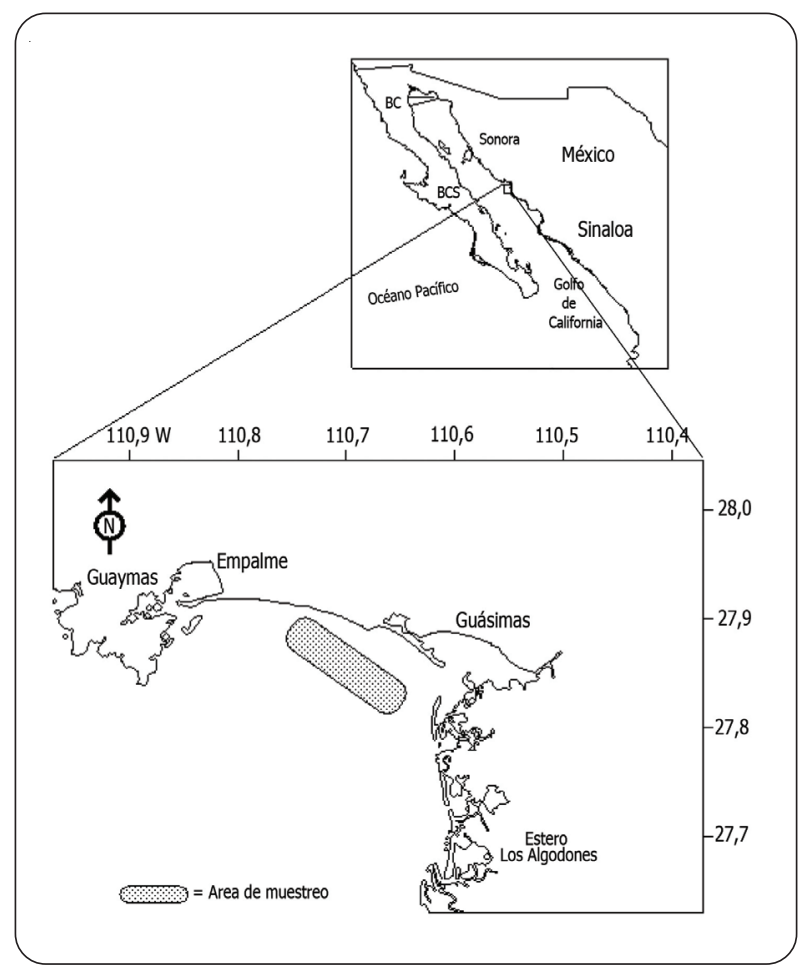

Figura 1. Área de estudio de Panopea globosa en la región central del Golfo de California, México / Study area of Panopea globosa in the central Gulf of California, Mexico
Múltivariado de El Niño (MEI) para todo el periodo. Los años en que ocurrieron eventos El Niño o La Niña, de acuerdo con el MEI, fueron seleccionados y se representaron en la gráfica de climatología, iniciando en el mes de mayo y finalizando en abril para identificar la mayor intensidad de los eventos El Niño o La Niña en el MEI y no como años calendario (enero-diciembre).

\section{DETERMINACIÓN Y VALIDACIÓN DE LA EDAD}

La edad de cada individuo se estimó utilizando la técnica de Shaul \& Goodwin (1982). La valva derecha de cada individuo fue cortada con una sierra de diamante de baja velocidad a través del centro de la placa de la charnela. La superficie de corte fue pulida y grabada con solución de ácido clorhídrico al 5\% para enjuagarse después de 15 min con agua destilada y secada. La impresión en el acetato se obtuvo presionando la película de acetato, suavizado con una gota de acetona, en la sección grabada de la placa de la charnela. Los anillos de crecimiento impresos en la laminilla de acetato fueron contados utilizando un microscopio Carl Zeiss ${ }^{\circledR}$. Cada laminilla se contó en dos lectores diferentes para confirmar la edad estimada (Fig. 2). Los datos de edad fueron comprobados por el personal del Laboratorio de Esclerocronología de la Estación Biológica del Pacífico de Nanaibo, Canadá utilizando la técnica de datación cruzada.

Figura 2. Anillos de crecimiento en las valvas de Panopea globosa. Los números indican la edad en años / Growth rings in the valves of Panopea globosa. Numbers indicate age in years

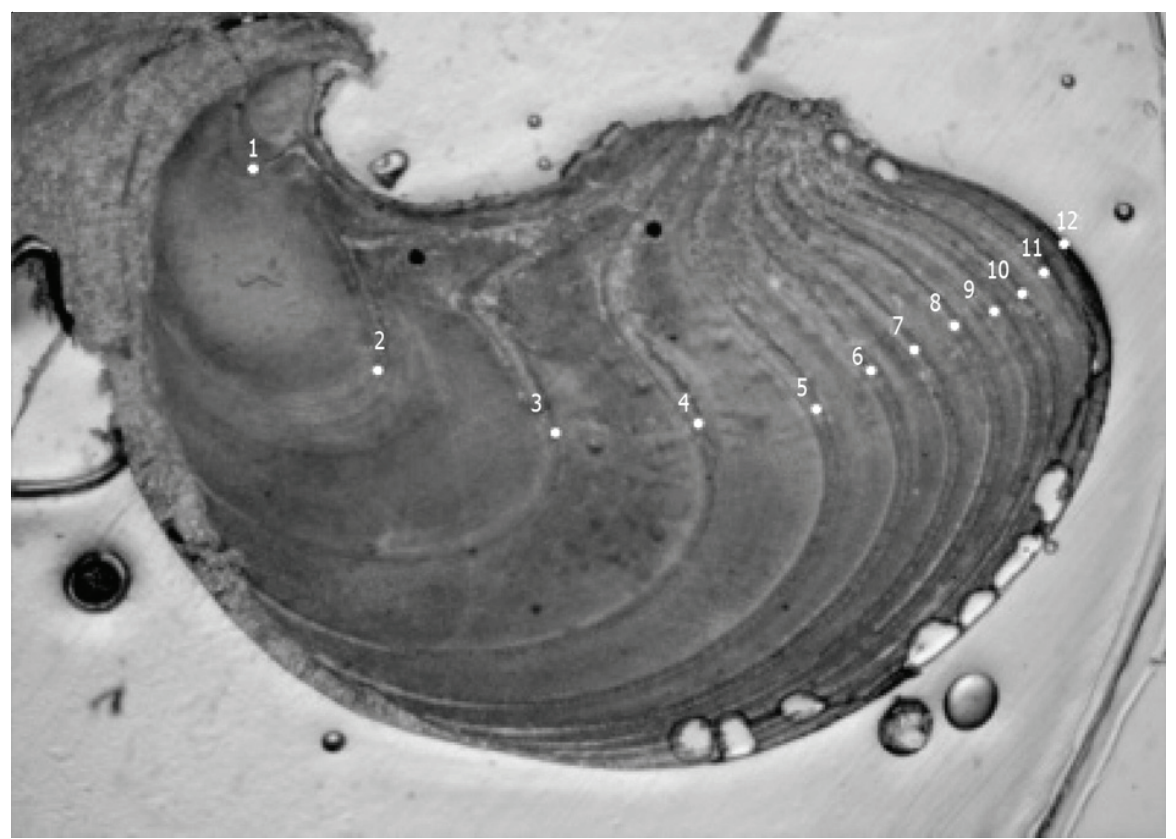


Fue necesario validar que las bandas de crecimiento observadas y contadas corresponden a incrementos anuales. Aunque Shaul \& Goodwin (1982) realizó esta validación para $P$. generosa, esto no se ha hecho para $P$. globosa. La técnica de 'datación cruzada' ha sido usada para obtener lecturas exactas de edad (validación de edad) en organismos longevos como peces y bivalvos bajo el supuesto que el medio ambiente sincroniza los incrementos del crecimiento y que por lo tanto produce (en organismos del mismo sitio) patrones consistentes de bandas estrechas y amplias a las que se les conoce como años firma (AF) y que pueden ser asignadas a un año específico (Black et al. 2008, Black 2009).

El conteo de anillos de crecimiento se realizó con el uso de un microscopio. Se puso especial atención en obtener conteos exactos y precisos. Se cuenta por primera vez las líneas hialinas de crecimiento de verano desde el extremo hacia el origen del umbo. Por cada muestra de conchas se trató de identificar (primero en los acetatos mejor hechos) los AF y asignarles un año específico. Las conchas de un banco o una localidad pueden presentar diferentes AF debido a los microclimas. Posteriormente, se realizó una segunda lectura usando los AF como referencia para confirmar la edad. La lógica de la exactitud consiste en que se conoce la fecha de recolecta y por lo tanto también se conoce el año que corresponde al anillo de crecimiento más externo, la identificación de los AF, permite determinar con mayor exactitud los años correspondientes a los anillos internos.

Con base a lo anterior, estamos suponiendo que si una concha con un número de anillos $A_{x}$, tiene un año marca $S_{x}$ (amplio o angosto) que se puede asignar a un año específico, y que si otra concha recolectada un año después con un número de anillos $A_{x+1}$ muestra el mismo año marca $S_{x}$ entonces se valida la aparición anual de los anillos (Black et al. 2008, Black 2009).

\section{ESTRUCTURA DE LONGITUD TOTAL DE CONCHA}

Una vez extraídas las almejas fueron transportadas al laboratorio a baja temperatura y procesadas inmediatamente. La longitud individual de la concha (distancia de la línea recta entre los márgenes anterior y posterior de la concha), se midió con calibres de Vernier (mm), siguiendo lo descrito por Aragón-Noriega et al. (2007). Posteriormente los datos fueron agrupados en intervalos de $10 \mathrm{~mm}$ para el análisis de la estructura de tallas.

\section{Crecimiento Corporal}

Los parámetros de crecimiento fueron determinados utilizando los datos de longitud-edad contando los anillos anuales. Las curvas de crecimiento de von Bertalanfy (VB) se aplicaron a todos los puntos de datos como la descrita por la ecuación:

$$
L_{t}=L_{\infty}\left(1-\exp \left(-k\left(t-t_{0}\right)\right)\right)+\varepsilon_{2}
$$

Donde: $t$ es la edad en años, $L_{t}$ es la longitud de la concha $(\mathrm{mm})$ a la edad $\mathrm{t}, L_{\infty}$ es la longitud media asintótica, $k$ es el coeficiente de crecimiento que determina la tasa de aumento o disminución en incrementos de longitud, $t_{0}$ sugiere la edad hipotética en la que el organismo habría sido longitud cero y $\varepsilon_{2} \approx N\left(0, \sigma_{1}^{2}\right)$ representa el error estándar. Los parámetros, $L_{\infty}, k$ y $t_{0}$ se calcularon utilizando el algoritmo de Levenberg-Marquardt, no lineal de mínimos cuadrados (Moré 1978), y la función objetivo fue la suma de la diferencia de cuadrados:

$$
\sum\left(L_{t}-L_{\infty} *\left(1-e^{-k\left(t-t_{0}\right)}\right)\right)^{2}
$$

\section{Mortalidad}

La mortalidad natural instantánea (M) se estimó con tres diferentes métodos, primero a través de la ecuación de Pauly (1980), por medio de la relación empírica de dos parámetros del crecimiento von Bertalanffy y la temperatura ambiental, como sigue:

$$
M=k^{0,65} \cdot L_{\infty}{ }^{-0,279} \cdot T_{c}^{0,463}
$$

Donde $M$ es la tasa de mortalidad natural anual instantánea (proporción por año) y $T_{c}$ es la temperatura promedio del hábitat (agua) que se mide en grados Celsius. Para este estudio se usó la temperatura mínima y máxima del periodo estudiado.

El segundo método fue utilizando la ecuación de longevidad que calcula la mortalidad mediante la ecuación:

$$
M=\frac{\ln (p)}{A}
$$

Donde $A$ es la edad máxima y $P$ la proporción de la población que se supone alcanza la edad máxima. El valor de $P=0,01$ ha sido utilizado para otras especies del género Panopea (Gribben \& Creese 2005, Calderón-Aguilera et al. 2010a) y también se usó en este análisis.

El tercer método fue a través del estimador de mortalidad de Chapman-Robson (CR) que calcula la mortalidad utilizando datos de captura y edad con la siguiente ecuación:

$$
C R=\ln \left(\frac{1+a-1 / n}{a}\right)
$$


Donde $a$ es la edad promedio y $n$ es el tamaño de muestra (Chapman \& Robson 1960). Según Gribben \& Creese (2005) es el método que menor sesgo presenta.

\section{Resultados}

\section{Temperatura SUPERFICIAL DEL MAR}

La temperatura media anual de la superficie del mar fue $24,2^{\circ} \mathrm{C}$ en la Bahía del Sol, Sonora, México (diciembre de 1981 a marzo de 2010). La variación estacional de la temperatura superficial del mar (TSM) fue calculada promediando mensualmente la TSM de diciembre de 1981 a marzo de 2010. El mínimo se presentó en febrero (18,0 \pm $\left.1,4^{\circ} \mathrm{C}\right)$ y el máximo en agosto $\left(30,1 \pm 1,1^{\circ} \mathrm{C}\right)$. Se observó una marcada variabilidad estacional (Fig. 3). El valor del MEI para el bimestre mayo/junio de 1988 a abril/mayo de 1989 fue de -1,1 (periodo más frío para nuestro estudio). Asimismo, el valor del MEI para el bimestre mayo/junio de 1997 a abril/mayo de 1998 fue de 2,5 (periodo más cálido para nuestro estudio). En la figura 3 se observa que esos periodos coinciden con los más fríos y más cálidos en cuanto a TSM. Es importante señalar que la mayor diferencia entre la TSM promedio, el periodo más cálido y el periodo más frío, se presenta en los meses de noviembre a marzo (Fig. 3).

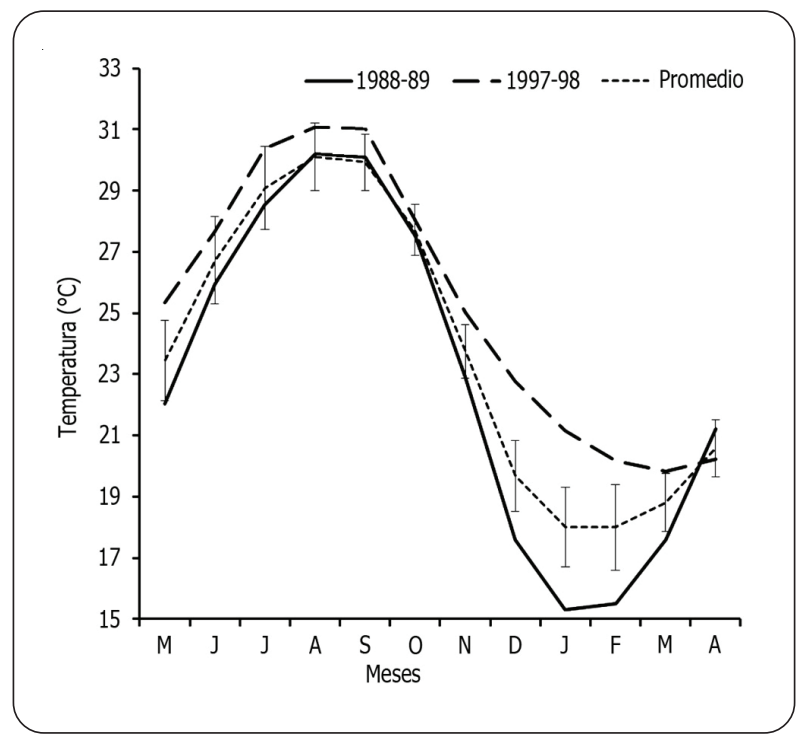

Figura 3. Promedio mensual de la serie de tiempo anual de temperatura superficial del mar de 1983 a 2010 en la región central del Golfo de California / Monthly average of annual time series of sea surface temperature from 1983 to 2010 in the central Gulf of California region

\section{VALIDACIÓN DE EDAD}

La fecha de extracción de la almeja fue de noviembre de 2008 a febrero de 2010. Se identificó cada concha con la fecha de extracción y se procedió a contar los anillos desde el extremo hasta el umbo. Se encontró en varias conchas una banda amplia que se repetía y fue determinada como el año 1998 que representó el año Niño de 19971998 (Fig. 4). A este AF (año firma) se le realizó un seguimiento, con el supuesto que una posterior concha con un número de anillos $A_{x}$, tiene el $\mathrm{AF}_{1998}$ (banda amplia) determinado en el año 1998. Con las conchas recolectadas un año después con el número de anillos $A_{x+1}$ y que presentaron el mismo $\mathrm{AF}_{1998}$ se determinó que las bandas observadas representan incrementos anuales en $P$. globosa.

\section{ESTRUCTURA DE EDAD Y DE LONGITUD TOTAL}

Las edades estimadas variaron de 2 a 27 años. La distribución de edad (Fig. 5) mostró que el grupo de 10 años fue la moda con 36 individuos. El siguiente grupo con más individuos capturados fue el de 12 años con 28 individuos y el de 8 años con 22 individuos capturados.

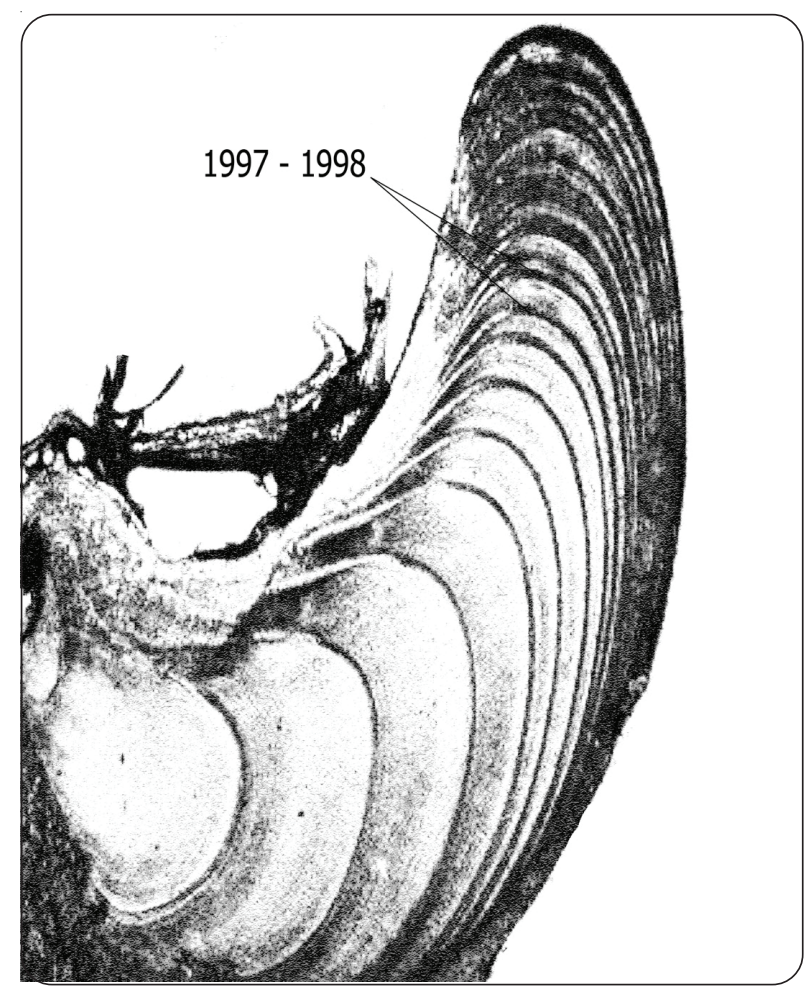

Figura 4. Anillos de crecimiento mostrando uno de los años firma (AF) para Panopea globosa / Growth rings as an example of signature year (AF) in Panopea globosa 


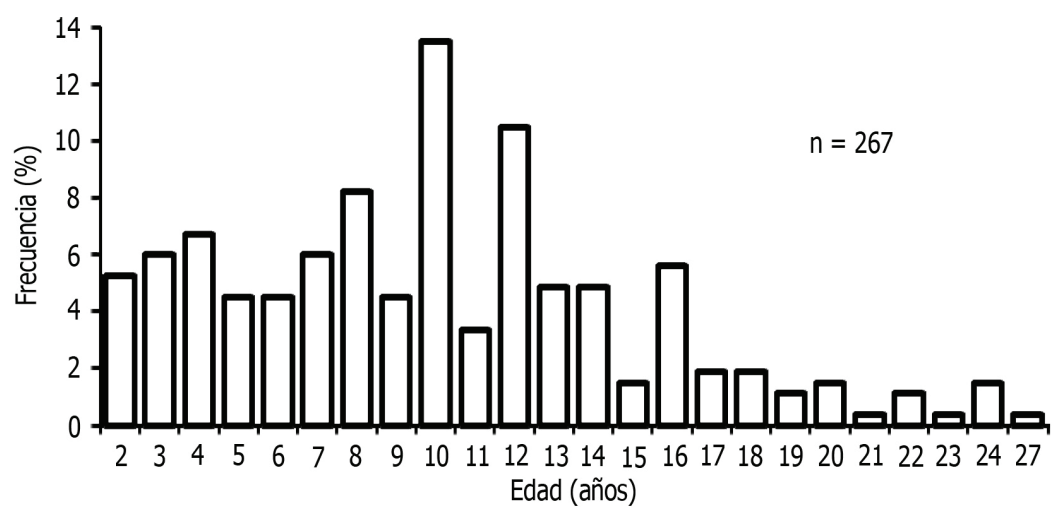

Figura 5. Histograma de estructura de edades para Panopea globosa / Histogram of age structure of Panopea globosa

Los promedios de longitud para las distintas clases anuales de $P$. globosa (Tabla 1) oscilaron entre los $66 \mathrm{~mm}$ y los 138 , mostrando la moda en la talla de $120 \mathrm{~mm}$ y seguida por las de 130 y $110 \mathrm{~mm}$. La longitud promedio ( \pm IC $\alpha 0,05)$ de la concha fue de $109.8 \pm 3 \mathrm{~mm}$ y las tallas mínimas y máximas fueron 40 y $198 \mathrm{~mm}$ respectivamente (Fig. 6).

\section{Crecimiento corporal}

Se ajustó el modelo de crecimiento de von Bertalanffy con el que se calculó una longitud asintótica $\left(L_{\infty}\right)$ de 122,86 $\mathrm{mm}$ y una tasa de crecimiento $(k)$ de 0,33 (Fig. 7). Se encontró que el crecimiento de la concha es rápido durante los 5 primeros años y, posteriormente disminuye considerablemente, siendo muy lento para los organismos mayores de 10 años.

Según la edad determinada se encontró que el individuo más longevo en esta zona fue de 27 años y midió $112 \mathrm{~mm}$ de largo. Por otro lado los individuos más jóvenes tenían 2 años de edad y midieron $66,2 \mathrm{~mm}$ (ver Tabla 1 para mayores detalles). Se resalta que el grupo de 10 años fue el más representado y también representó el grupo cuya longitud promedio fue la más grande $(138,7 \mathrm{~mm})$.

\section{Mortalidad}

Con los parámetros estimados de crecimiento y con el valor promedio de temperatura superficial del mar de todo el periodo $\left(24,2^{\circ} \mathrm{C}\right)$ se calculó la mortalidad natural en 0,546 . Utilizando el valor de mínima temperatura $\left(17,2^{\circ} \mathrm{C}\right)$ la mortalidad fue de 0,467 y con el valor máximo de temperatura $\left(31,2^{\circ} \mathrm{C}\right)$ se encontró una mortalidad de 0,616 . Con la ecuación de longevidad se calculó la mortalidad en 0,171 y con el de Chapman-Robson la mortalidad se estimó en 0,079 .
Tabla 1. Número y frecuencia de especímenes utilizados de Panopea globosa para el cálculo promedio de longitud en distintas edades ( 2 a 27 años) / Number and frequency of specimens of Panopea globosa analyzed for average length at different estimated ages ( 2 to 27 years)

Edad Longitud valvar Desviación Número Frecuencia (años) Promedio (mm) estándar de datos (\%)

\begin{tabular}{|c|c|c|c|c|}
\hline 2 & 66,21 & 9,6 & 14 & 5,2 \\
\hline 3 & 74,13 & 18,7 & 16 & 6,0 \\
\hline 4 & 76,98 & 20,2 & 18 & 6,7 \\
\hline 5 & 94,92 & 18,5 & 12 & 4,5 \\
\hline 6 & 96,42 & 19,2 & 12 & 4,5 \\
\hline 7 & 108,75 & 13,8 & 16 & 6,0 \\
\hline 8 & 113,23 & 16,3 & 22 & 8,2 \\
\hline 9 & 118,08 & 16,1 & 12 & 4,5 \\
\hline 10 & 138,67 & 22,3 & 36 & 13,5 \\
\hline 11 & 117,67 & 17,4 & 9 & 3,4 \\
\hline 12 & 116,29 & 12,1 & 28 & 10,5 \\
\hline 13 & 119,31 & 7,4 & 13 & 4,9 \\
\hline 14 & 119,15 & 13,1 & 13 & 4,9 \\
\hline 15 & 119,15 & 11,7 & 4 & 1,5 \\
\hline 16 & 122,67 & 23,4 & 15 & 5,6 \\
\hline 17 & 110,80 & 21,7 & 5 & 1,9 \\
\hline 18 & 100,60 & 12,6 & 5 & 1,9 \\
\hline 19 & 128,33 & 20,8 & 3 & 1,1 \\
\hline 20 & 129,25 & 21,3 & 4 & 1,5 \\
\hline 21 & 76,00 & & 1 & 0,4 \\
\hline 22 & 109,33 & 10,1 & 3 & 1,1 \\
\hline 23 & 97,00 & & 1 & 0,4 \\
\hline 24 & 109,00 & 18,6 & 4 & 1,5 \\
\hline 27 & 112,00 & & 1 & 0,4 \\
\hline
\end{tabular}


Figura 6. Histograma de estructura de longitud valvar para Panopea globosa / Histogram of valvar length structure of Panopea globosa

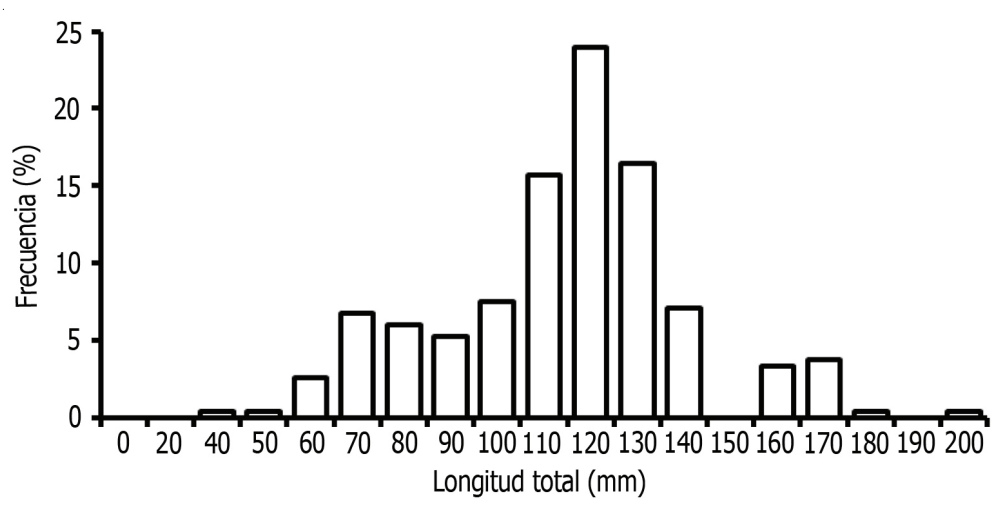

Figura 7. Modelo de estimación de crecimiento de Von Bertalanffy para Panopea globosa / The Von Bertalanffy' growth model fit to Panopea globosa

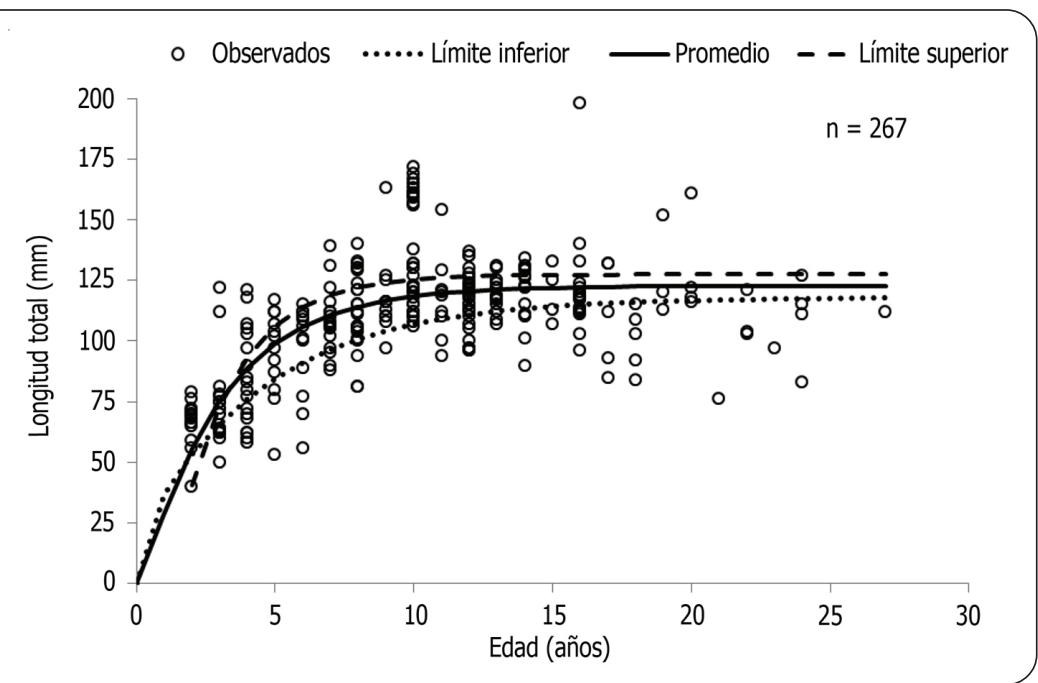

\section{Discusión}

Los resultados del presente estudio son los primeros en analizar crecimiento de Panopea globosa utilizando el método de identificación y conteo de anillos de crecimiento. Los resultados de esta técnica con las especies del mismo género sugieren que los anillos de crecimiento encontrados en P. globosa son anuales. Lo anterior fue validado con la técnica de datación cruzada (Black et al. 2008, Black 2009). En P. globosa los anillos internos de crecimiento de la concha se muestran como una región angosta y traslúcida formada en el invierno y otra region amplia y opaca que se forma de primavera a otoño. Los estudios que se han realizado en otras almejas del mismo género (Morsán \& Ciocco 2004) sugieren que los patrones traslúcidos son determinados por la actividad metabólica asociada con cambios en la temperatura del agua del fondo marino. En este sentido Shaul \& Goodwin (1982) explicaron que la línea de crecimiento que se forma en $P$. generosa es por la disolución del carbonato de calcio debido al metabolismo anaeróbico durante su periodo de inactividad. La almeja de sifón del hemisferio norte $P$. generosa (Shaul \& Goodwin 1982), así como la del hemisferio sur P. abbrevita (Morsán \& Ciocco 2004) retraen su sifón permaneciendo en una aperente condición de inactividad. Considerando que $P$. globosa, distribuida en la parte central del Golfo de California y congenere de las especies citadas, es probable que tenga un comportamiento similar a éstas.

Además de los métodos directos para estimar el crecimiento en bivalvos, existen otros indirectos siendo el más usado el de contar las bandas de crecimiento de la concha (Richardson \& Walker 1991), la técnica de contar los anillos de crecimiento para la estimación de la edad de almeja de sifón en el mundo fue validado por Shaul \& 
Goodwin (1982) y se utilizó para determinar la edad de Panopea generosa en la Columbia Británica (Bureau et al. 2002, 2003) y en Washington (Goodwin \& Shaul 1984), para $P$. zelandica en Nueva Zelanda (Breen et al. 1991), y para P. abbreviata en Argentina (Morsán \& Ciocco 2004). La lectura de anillos ha sido reforzada mediante técnicas de datación cruzada, por medio de dendrocronología, que busca patrones de crecimiento sincrónico de una población objeto de muestreo y utiliza años 'firma' (AF) de una referencia cruzada entre los especímenes de una muestra (Black 2009). En las almejas analizadas del Golfo de California se encontró que esta técnica puede ser utilizada para las lecturas de anillos y representa una buena medida de crecimiento anual. Los llamados AF se reflejan en las almejas si algún evento climático favorable o desfavorable se presenta en el hábitat. El Niño Oscilación del Sur (ENOS) ocurrido en 1983-1984 parece tener un efecto favorable para $P$. generosa de Baja California (Calderón-Aguilera et al. 2010a). Las observaciones de estos autores indican que en la almeja de sifón el crecimiento es más rápido durante el período caluroso, y el ENOS mantiene condiciones más cálidas en el agua del mar. La presencia de El Niño de 1997-1998 (Pérez-Arvizu 2009) puede ser la explicación de que el grupo de 10 años fue el más representado en este trabajo, lo que sugiere mecanismos más complejos que explican la variabilidad del reclutamiento.

Se puede considerar que la deposición de anillos de crecimiento interno se produce durante los meses de invierno en el Golfo de California y por lo tanto pueden utilizarse para las almejas de esta región. Por ejemplo, para Panopea generosa de Canadá y Washington se determinó con exactitud que las bandas de crecimiento son anuales (Shaul \& Goodwin 1982). Lo mismo fue comprobado para P. abreviatta de Argentina (Morsán \& Ciocco 2004) y para $P$. zelandica de Nueva Zelanda (Gribben \& Creese 2005).

La estimación de los parámetros de crecimiento individual halló una longitud asintótica total de la concha de 122,9 mm. Esta longitud asintótica está por debajo del intervalo encontrado para Panopea generosa en la Columbia Británica que fue reportado de 129 a $147 \mathrm{~mm}$ (Bureau et al. 2002, 2003, Campbell \& Ming 2003) y la encontrada en el Estado de Washington que fue de 132 a $173 \mathrm{~mm}$ (Hoffmann et al. 2000). Por otro lado, la longitud asintótica de $P$. globosa es más grande que la de otras especies de almeja de sifón del hemisferio sur: $106 \mathrm{~mm}$ longitud total de la concha para $P$. abbreviata (Morsán \& Ciocco 2004) y $116 \mathrm{~mm}$ para P. zelandica (Gribben \&
Creese 2005). En el análisis de las tallas crudas encontramos un individuo de $40 \mathrm{~mm}$ como la talla mínima capturada y la talla máxima de $198 \mathrm{~mm}$. Es importante señalar que aunque se presenten organismos de longitudes tan grandes lo que procede reconocer es la probabilidad tan baja de que aparezcan estas tallas extremas y que el análisis de datos considera el comportamiento poblacional. Aquí también es necesario comentar que en otras especies de género se ha observado que las longitudes son ampliamente variables a una misma edad y que el organismo más longevo no es el de la longitud más grande. La probabilidad de encontrar a un organismo longevo siempre es muy baja, tanto así que para estimaciones de mortalidad se ha tomado como 0,01 la probabilidad de encontrar al organismo más longevo (Gribben \& Creese 2005, Caderón-Aguilera et al. 2010a). Morsán \& Ciocco (2004) encontraron el organismo más longevo de $P$. abbreviata de casi 40 años de edad con una longitud de $80 \mathrm{~mm}$, mientras que un organismo de 20 años media $120 \mathrm{~mm}$. Breen et al. (1991) encontraron el organismo más longevo de $P$. zelandica con una edad de 25 años y $105 \mathrm{~mm}$ de longitud y un organismo de 15 años con $135 \mathrm{~mm}$ de longitud total. De igual forma Caderón-Aguilera et al. (2010a) encontraron el organismo más longevo de $P$. generosa de 96 años con $140 \mathrm{~mm}$ y un organismo de 20 años con $160 \mathrm{~mm}$. Por lo tanto no es sorprendente y mucho menos un posible error de evaluación el que este comportamiento se haya presentado en P. globosa del Golfo de California.

Otro aspecto que pudiera ser cuestionable es el sesgo de longitudes pequeñas por la extracción selectiva de organismos mayores por la pesca, pero en esta especie no puede ser seleccionada la longitud del organismo antes de ser extraída del subsuelo marino. Hasta el momento no se ha logrado establecer una relación entre la marca del sifón y la longitud total del organismo. Para su extracción el buzo observa una marca que deja el sifón al contraerse y extrae la almeja, pero nunca se puede seleccionar la longitud. Gribben \& Creese (2005) proponen para Panopea zelandica una relación entre el ancho de la marca y la longitud, pero a nivel de pesca comercial esto ha sido extremadamente difícil lograrlo. Las tallas encontradas en este estudio no fueron el resultado de pesca selectiva por tallas sino el estado natural de la población.

La talla mínima legal establecida para la pesquería de la especie en México es de $130 \mathrm{~mm}$ (Calderón-Aguilera et al. 2010a). Sin embargo, nuestros resultados mostraron que la longitud asintótica fue $<130 \mathrm{~mm}$, y por lo tanto el límite de tamaño establecido por las autoridades, parece 
ser demasiado alto. En el presente estudio se encontró que el $31 \%$ de los organismos capturados fueron iguales o superiores a esta longitud. Otro aspecto importante a considerar en la pesquería es que una almeja de sifón adulta es incapaz de volver a enterrarse ya que al ser retirada del sustrato pierde la protección del mismo y no cuenta con su apéndice de excavación, el cual pierde en su edad adulta (Feldman et al. 2004). En la actualidad con la talla mínima legal establecida, los pescadores descartan los organismos de tallas menores, ocasionando un gran desperdicio de recursos. Con base en la toma de muestras biológicas de este estudio, se estimó que la mortalidad ocasionada por este hecho podría ser de aproximadamente el 38\%. La talla crítica o mínima legal deberá estar basada en la talla de primera madurez, sin embargo los estudios relativos a edad o talla de primera madurez aún no se han realizado. Debido a que cada especie presenta diferentes parámetros de crecimiento, tampoco es prudente utilizar las tallas de primera madurez de sus congéneres como $P$. generosa o P. zelandica (Campbell \& Ming 2003, Gribben $\&$ Creese 2003)

Hoffmann et al. (2000) afirmaron que el parámetro de crecimiento, $k$, en el modelo de von Bertalanffy es un factor clave para proponer cuotas de captura adecuadas a esa población. En este estudio se encontró que el valor de $k$ fue de 0,33 y es mayor a los estimados previamente para otras especies del género: Panopea generosa en Washington $(0,113$ a 0,235$)$ Hoffmann et al. (2000), P. generosa en Canadá $(0,146$ y 0,189$)$ Campbell \& Ming (2003) y P. abbreviata $(0,183)$ (Morsán \& Ciocco 2004) y P. zelandica $(0,16)$ (Breen et al. 1991) en el Hemisferio sur. Se conoce que el ajuste del modelo de crecimiento a los datos depende de la estructura demográfica de la población estudiada. Normalmente los valores 'grandes' de $k$ se encuentran cuando las poblaciones objetivo se componen de individuos más jóvenes (Hoffmann et al. 2000). Lo que sugiere que la población de P. globosa de la parte central del Golfo de California es de reciente asentamiento en respuesta a un evento ambiental favorable.

La mortalidad natural estimada con la ecuación de longevidad y la de Chapman-Robson están entre los valores reportados para otros congéneres como la Panopea generosa del Pacífico oriental. Sin embargo la mortalidad estimada con la ecuación empírica de Pauly (1980) es irreal para esta especie. El mismo Pauly (1980) lo indica como una ecuación adecuada para peces y como se ha demostrado en este estudio no puede ser extendida para P. globosa. La temperatura tiene un impacto en el crecimiento y se ha detectado en la amplitud de las bandas anuales de crecimiento, pero no es un buen indicador para determinar mortalidad por medio de la ecuación empírica de Pauly (1980).

En resumen, esta primera información biológica relativa a los parámetros de crecimiento de la almeja de sifón Panopea globosa en la parte central del Golfo de California, aporta evidencia de que la talla mínima legal para la pesca de la almeja de sifón en México es demasiado grande $(130 \mathrm{~mm})$. Por lo que dicha estrategia de gestión parece inadecuada y podría ocasionar pérdidas económicas para los productores. Los parámetros de crecimiento y la elevada mortalidad observados coinciden a los calculados en otras especies del género en organismos jóvenes, por lo que se considera que la población estudiada se asentó recientemente, o bien puede ser un stock que procede de un reclutamiento que fue favorecido por condiciones ambientales adecuadas a sus requerimientos.

\section{Agradecimientos}

GCL agradece al CONACYT por la beca otorgada para realizar sus estudios de posgrado. Edgar Alcántara-Razo colaboró con el trabajo en laboratorio y campo. El proyecto fue financiado por pescadores privados y sociales del Sur de Sonora. La cooperativa Ricardo Loreto aportó los buzos de apoyo. Los revisores anónimos fueron de gran apoyo para mejorar el presente documento.

\section{LITERATURA CITADA}

Aragón-Noriega EA, J Chávez-Villalba, PE Gribben, E Alcántara-Razo, AN Maeda-Martínez, EM ArámbulaPujol, AR García-Juárez \& R Maldonado-Amparo. 2007. Morphometric relationships, gametogenic development and spawning of the geoduck clam Panopea globosa (Bivalvia: Hiatellidae) in the Central Gulf of California. Journal of Shellfish Research 26(2): 423-431.

Arámbula-Pujol EM, AR García-Juárez, E Alcantára-Razo \& EAAragón-Noriega 2008. Aspectos de biología reproductiva de la almeja de sifón Panopea globosa (Dall, 1898) en el Golfo de California. Hidrobiológica 18(2): 89-98

Black BA. 2009. Climate-driven synchrony across tree, bivalve, and rockfish growth-increment chronologies of the northeast Pacific Marine Ecology Progress Series 378: 37-46.

Black BA, DC Gillespie, SE McLellan \& CM Hand. 2008. Establishing highly accurate production-age data using the tree-ring technique of crossdating: a case study for Pacific geoduck (Panopea abrupta). Canadian Journal of Fisheries and Aquatic Sciences 65: 2572-2578. 
Bradbury A \& JV Tagart. 2000. Modeling geoduck, Panopea abrupta (Conrad 1849) population dynamics. II. Natural mortality and equilibrium yield. Journal of Shellfish Research 19: 63-70.

Breen PA, C Gabriel \& T Tyson. 1991. Preliminary estimates of age, mortality, growth, and reproduction in the hiatellid clam Panopea zelandica in New Zealand. New Zealand Journal of Marine and Freshwater Research 25: 231-237.

Bureau D, W Hajas, NW Surry, CM Hand, G Dovey \& A Campbell. 2002. Age, size structure, and growth parameters of geoducks (Panopea abrupta Conrad, 1849) from 34 locations in British Columbia sampled between 1993 and 2000. Canadian Technical Reports of Fisheries and Aquatic Sciences 2413: 1-84.

Bureau D, W Hajas, CM Hand \& G Dovey. 2003. Age, size structure and growth parameters of geoducks (Panopea abrupta, Conrad 1849) from seven locations in British Columbia sampled between 2001 and 2002. Canadian Technical Reports of Fisheries and Aquatic Sciences 2494: $1-29$.

Calderón-Aguilera LE, EA Aragón-Noriega, CM Hand \& VM Moreno-Rivera. 2010a. Morphometric relationships, age, growth and mortality of the geoduck clam Panopea generosa, along the Pacific coast of Baja California, Mexico. Journal of Shellfish Research 29(2): 319-326.

Calderón-Aguilera LE, EA Aragón-Noriega, H ReyesBonilla, CG Paniagua-Chávez, AE Romo-Curiel \& VM Moreno-Rivera. 2010b. Reproduction of the Cortes geoduck Panopea globosa (Bivalvia: Hiatellidae) and its relationship with temperature and ocean productivity. Journal of Shellfish Research 29: 131-141.

Campbell A \& MD Ming. 2003. Maturity and growth of the Pacific geoduck clam, Panopea abrupta, in Southern British Columbia. Canadian Journal of Shellfish Research 22: 85-90.

Chapman DG \& DS Robson. 1960. The analysis of a catchcurve. Biometrics 16: 354-368.

Feldman K, B Vadopalas, D Armstrong, C Friedman, R Hilborn, K Naish, J Orensanz \& J Valero. 2004. Comprehensive literature review and synopsis of issues relating to geoduck (Panopea abrupta) ecology and aquaculture production, $140 \mathrm{pp}$. Washington State Department of Natural Resources, Olympia.

Goodwin CL \& W Shaul. 1984. Age, recruitment and growth of the geoduck clam (Panopea generosa, Gould) in Puget Sound, Washington. Washington Department of Fisheries Technical Report, Olympia 215: 1-29.

Gribben PE \& RG Creese. 2003. Protandry in the New Zealand geoduck, Panopea zelandica (Mollusca, Bivalvia). Invertebrate Reproduction and Development 44: 119-129.

Gribben PE \& RG Creese. 2005. Age, growth, and mortality of the New Zealand geoduck clam, Panopea zelandica (Bivalvia: Hiattellidae) in two North Island populations. Bulletin of Marine Science 77: 119-135.
Hoffmann A, A Bradbury \& CL Goodwin. 2000. Modelling geoduck, Panopea abrupta (Conrad 1849) population dynamics growth. Journal of Shellfish Research 19: 57-62.

Khan AS. 2006. Sustainability challenges in the geoduck clam fisheries of British Columbia: Policy perspectives. Coastal Management Journal 34(4): 443-453.

Mora C, RA Myers, M Coll, S Libralato, TJ Pitcher, RU Sumaila, D Zeller, R Watson, KJ Gaston \& B Worm. 2009. Management Effectiveness of the World's Marine Fisheries. PLoS Biol 7(6): e1000131. <doi:10.1371/ journal.pbio.1000131>

Moré JJ. 1978. The Levenberg-Marquardt algorithm: implementation and theory. En: Watson GA (ed). Lecture notes in mathematics 630: 105-116. Springer-Verlag, Berlin.

Morsán E \& N Ciocco. 2004. Age and growth model for the southern geoduck, Panopea abbreviata, off Puerto Lobos (Patagonia, Argentina). Fisheries Research 69: 343-348.

Orensanz JM, CM Hand, AM Parma, J Valero \& R Hilborn. 2004. Precaution in the harvest of Methuselah's clams the difficulty of getting timely feedback from slow-paced dynamics? Canadian Journal of Fisheries and Aquatic Sciences 61: 1355-1372.

Pauly D. 1980. On the interrelationships between natural mortality, growth parameters, and mean environmental temperature in 175 fish stocks. ICES Journal of Marine Science 39: 175-192.

Pauly D. 1996. ITQ: The assumptions behind a meme. Reviews in Fish and Fisheries Biology 6: 109-112.

Pérez-Arvizu EM. 2009. Caracterización del ecosistema del Alto Golfo de Califonia por medio de percepción remota y su relación con la captura de camarón azul Litopenaeus stylirostris y curvina golfina Cynoscion othonopterus. Tesis de Maestría, CIIDIR-IPN Unidad Sinaloa, Guasave, Sinaloa, $106 \mathrm{pp}$.

Richardson CA \& P Walker. 1991. An analysis of the age structure of the hard shell clam Mercenaria mercenaria from acetate peel replicas of shell sections. ICES Journal of Marine Science 50: 493-500.

Shaul W \& L Goodwin. 1982. Geoduck (Panopea generosa: Bivalvia) age as determined by internal growth lines in the shell. Canadian Technical Reports of Fisheries and Aquatic Sciences 39: 632-636.

Sloan NA \& SMC Robinson. 1984. Age and gonad development in the geoduck clam Panopea abrupta (Conrad) from southern British Columbia. Canadian Journal of Shellfish Research 4: 131-137.

Tijaro-Rojas R \& H Salgado-Cabrera. 2007. Efectos de largo plazo de instrumentos de regulación pesquera: Caso de la pesquería del jurel Trachurus murphyi (Nichols, 1920) en la zona centro sur de Chile. Boletín de Investigaciones Marinas y Costeras 36: 49-62. 